\title{
Inverse association between negative symptoms and body mass index in chronic schizophrenia
}

\section{Running title: Negative symptoms and body mass index in schizophrenia}

Mezquida $\mathrm{G}^{1}$, Savulich $\mathrm{G}^{2}$, Garcia-Rizo C ${ }^{1,3,4}$, Garcia-Portilla MP ${ }^{3,5}$, Toll A ${ }^{3,6,7}$, GarciaAlvarez L ${ }^{3}$, Bobes $\mathrm{J}^{3,5}$, Mané A ${ }^{3,6,7}$, Bernardo $\mathrm{M}^{1,3,4,8}$ Fernandez-Egea $\mathrm{E}^{2,3,9}$ *

1 Barcelona Clinic Schizophrenia Unit, Neuroscience Institute, Hospital Clinic of Barcelona, Spain

2 Department of Psychiatry and Behavioural and Clinical Neuroscience Institute, University of Cambridge, United Kingdom

3 Centro de Investigación Biomédica en Red de Salud Mental (CIBERSAM)

4 Institut d'Investigacions Biomèdiques August Pi i Sunyer (IDIBAPS). Barcelona. Spain

5 Department of Psychiatry, University of Oviedo, Spain

6 Institut de Neuropsiquiatria i Adiccions, Parc de Salut Mar, Barcelona, Spain

7 Fundació Hospital del Mar Medical Research Institute (IMIM), Barcelona, Spain

8 Department of Medicine, University of Barcelona, Spain.

9 Cambridgeshire and Peterborough NHS foundation Trust, United Kingdom

\author{
* Corresponding Author: \\ Emilio Fernandez-Egea: \\ Clozapine Clinic | Cambriddgeshire and Peterborough NHS Foundation Trust \\ 128 Tenison Road | Cambridge, CB1 2DP \\ Email: ef280@cam.ac.uk \\ Tel. No. 00441223516969 | Fax. No. 00441223516967
}

\author{
Word Counts: $\mathbf{3 0 2 5}$ \\ Abstract: 250 \\ Tables: 2 \\ Figures: 1 \\ References: 38
}




\begin{abstract}
Background: We investigated whether negative symptoms, such as poor motivation or anhedonia, were associated with higher body mass index (BMI) in stable patients with schizophrenia chronically treated with antipsychotic medication.

Methods: 62 olanzapine- or clozapine-treated patients with illness duration of at least four years were selected from an international multicenter study on the characterization of negative symptoms. All participants completed the Brief Negative Symptom Scale (BNSS) and the Positive and Negative Syndrome Scale (PANSS). Bivariate correlations between BMI and negative symptoms (BNSS) were explored, as well as multiple regression analyses. We further explored the association of two principal component factors of the BNSS and BMI. Subsidiary analyses re-modeled the above using the negative symptoms subscale of the PANSS and the EMSLEY factor for negative symptoms for convergent validity.

Results: Lower negative symptoms (BNSS score) were associated with higher BMI ( $r=-.31$; $p=.015$ ). A multiple regression analysis showed that negative symptoms (BNSS score) and age were significant predictors of BMI $(p=0.037)$. This was mostly driven by the motivation/pleasure factor of the BNSS. Within this second factor, BMI was negatively associated with anhedonia $(r=-.254 ; p=.046)$ and asociality $(r=-.253 ; p=.048)$, but not avolition $(r=-.169$; $p=.188)$. EMSLEY score was positively associated with BNSS $(r=.873, p<.001)$, but negatively associated with BMI $(r=-.308 ; p=.015)$. The association between PANSS and BMI did not reach significance $(r=-224, p=.080)$.

Conclusions: We conclude that lower negative symptoms were associated with higher BMI (assessed using both the BNSS and EMSLEY) in chronic stable schizophrenia patients, mostly due to lower anhedonia and asociality levels.
\end{abstract}

Key words: Negative Symptoms, Body Mass Index, Schizophrenia, Brief Negative Symptom Scale, Clozapine 


\section{Introduction}

Strategies for preventing weight gain, one concern often reported by individuals receiving treatment in the early phases of psychosis, have been partially successful. However, weight loss interventions are more limited in chronic schizophrenia compared with other phases of the illness and in the general population, despite a higher prevalence of obesity (Royal College of Psychiatrists, 2014, Zimbron et al., 2016).

Increased incidence of obesity in schizophrenia has been grossly attributed to antipsychotic medication-induced appetite, although a dose-dependent relationship has not been supported (Simon et al., 2009). This view contrasts with the multi-factorial approach of obesity applied to non-psychiatric populations, which considers a variety of genetic, epigenetic and behavioral factors (Ziauddeen et al., 2015). Current models for predicting antipsychotic medicationinduced weight gain are unreliable (Fernandez-Egea et al., 2011) and there remains a need for identifying additional factors that might play a significant role in the cause and maintenance of weight gain leading to obesity in chronic schizophrenia. Intra-uterine stress (Ziauddeen et al., 2016) and genetic factors (Reynolds, 2012), for example, have already been shown to have an influence. Furthermore, it is likely that obesity in chronic schizophrenia has different predictors compared to other phases of the illness, due in part to variations in medication side effects, more persistent symptomatology and social consequences of the illness.

Negative symptoms severity has been intuitively associated with greater weight gain (Koga \& Nakayama, 2005), prompting speculation that problems with motivation underlie physical inactivity in patients with schizophrenia. However, evidence in support of this is scarce (Janney et al., 2013) and has only been provided by studies in populations with medication switching (Hermes et al., 2011) and during the onset of the illness (Pérez-Iglesias et al., 2014), when changes in psychopathology and body weight are more severe. Understating the role of persis- 
tent negative symptoms in chronic stable patients with schizophrenia may differ from those with a recent diagnosis, as antipsychotic medication-induced weight gain is known to plateau after three to five years of continuous treatment (Bushe et al., 2013). One study investigating weight gain in patients with chronic schizophrenia found that higher negative symptoms were associated with lower body mass index (Chen et al., 2014). If more severe negative symptoms predict obesity, then behavioural interventions for patients with chronic schizophrenia might be more successful if modules for reducing negative symptoms, such as motivation and anhedonia, are implemented (e.g. promoting physical exercise; increasing social interaction).

We aimed to test whether high levels of negative symptoms were associated with increased body mass index (BMI) in a multi-center international study on the characterization of negative symptoms in chronic schizophrenia. We included patients with at least four years of continuous prescribed antipsychotic treatment with clozapine or olanzapine, the two leading obesogenic antipsychotics (Leucht et al., 2013), in order to reduce medication heterogeneity after the peak risk of weight gain. For the assessment of negative symptoms, the Brief Negative Symptoms Scale (BNSS) and the Positive and Negative Syndrome Scale (PANSS) were used (both English (Kirkpatrick et al., 2011, Kay et al., 1987) and Spanish (Mané et al., 2014, Peralta \& Cuesta, 1994) versions). As secondary aims, we explored whether subscales of the BNSS and the more traditionally used PANSS predicted BMI. 


\section{Material and Method}

\subsection{Study sample}

The present sample was selected from a multi-centre cross sectional study of the characterization of negative symptoms in chronic schizophrenia, which had four primary aims, including investigation of the role of negative symptoms in metabolic disease.

The study recruitment dates were May 2015 to May 2016 across four centers, which included the Clozapine Clinic at the Cambridgeshire and Peterborough NHS Foundation Trust (Cambridge) and three in Spain: one in Oviedo (Oviedo) and two in Barcelona (Hospital del Mar (HMar) and Hospital Clinic (HClinic)). The present study is a continuation of a prior collaboration between sites for the translation and adaptation of the BNSS in Spanish (Mané et al., 2014), but all cases analyzed here have not been reported elsewhere. All procedures contributing to this work comply with the ethical standards of the relevant national and institutional committees on human experimentation and with the Helsinki Declaration of 1975, as revised in 2008 .

\subsection{Inclusion and exclusion criteria}

The multicenter study included patients with a diagnosis of schizophrenia (ICD-10 criteria) at any stage of illness. For this particular analysis, we included only those patients who were prescribed with clozapine or olanzapine, the two medications mostly associated with weight gain (Leucht et al., 2013a). An additional inclusion criteria was at least four years of antipsychotic treatment. All augmentation strategies were allowed, except for clozapine-aripiprazole, as we previously reported that this combination is associated with weight loss (Zimbron et al., 2016). No other exclusion criteria were applied. 


\subsection{Assessments}

All participants were evaluated with psychopathology scales as well as morphometric measures including a full medical and psychiatric history. The prescribed daily doses of antipsychotic medications were converted to an estimated equivalent amount of chlorpromazine in accordance with the international consensus (Gardner et al., 2010).

Morphometric evaluation included measurements of height, weight, diastolic and systolic blood pressure and heart rate during resting state. BMI was calculated using the standard formula $\left(\mathrm{BMI}=\right.$ weight $(\mathrm{kg}) /$ height $\left.^{2}\left(\mathrm{~m}^{2}\right)\right)$.

Psychopathology scales included the BNSS (Mané et al., 2014, Kirkpatrick et al., 2011) and PANSS (Kay et al., 1987, Peralta \& Cuesta, 1994), the Calgary Depression Scale (CDS) (Addington et al., 1990) and cognitive scales, the latter not included in the present study. To ensure interrater reliability between centres, each clinician (a total of five psychiatrists and two clinical psychologists) attended a training workshop to learn the administration and scoring of all the clinical tools used in the study (BNSS, PANSS and CDS).

\subsection{Use of psychopathology scales BNSS and PANSS.}

We used the total BNSS score calculated from all thirteen items (total BNSS). Two major components have been defined from this scale (Strauss et al., 2012): Factor 1 reflects an Emotional Expressivity dimension (PC1 BNSS), consisting of the Blunted Affect, Alogia, and Lack of Normal Distress subscales (items 4, 9, 10, 11, 12, 13); and Factor 2 reflects a Motivation and Pleasure dimension (PC2 BNSS), consisting of the Anhedonia, Avolition, and Asociality subscales (items 1, 2, 3, 5, 6, 7, 8). Some authors have argued that the BNSS comprises three, instead of two components (Garcia-Portilla et al., 2015) and that the alogia subscales (items 12 and 13) should also be considered as an independent component. For the purposes of this 
study, we first used the more widely accepted two components, but also explored the threecomponent view for comprehensiveness.

We used items P1 to P7 for the positive symptoms of the PANSS (Pos_PANSS). For negative symptoms, we used two different measures: the classical approach (sum of items N1 to N7, termed Neg PANSS), and the EMSLEY factor for negative symptoms (sum of items N1, N2, N3, N4, N6, GP7 and GP13 and GP16, termed Emsley PANSS (Emsley et al., 2003)). We chose these two factors because the PANSS negative subscale (N1 to N7) also contains pure cognitive symptoms (i.e. N5 and N6) and is thus less specific to negative symptoms. In addition, the BNSS and Neg_PANSS are also different measures of negative symptoms. For example, Neg_PANSS is based mostly on observed behavior, whereas the BNSS includes internal experiences as a factor of negative symptoms severity.

Before the introduction of newer and more valid scales, such as the BNSS, a common practice was the use of derived factors, which more accurately captured negative symptoms. In this sense, the negative symptoms Emsley PANSS factor includes three items related to avolition and associality experiences, and removes two items considered 'purely cognitive'.

\subsection{Statistical analyses}

Zero-order bivariate correlations between total BNSS scores and BMI were explored using Person's correlation. Multiple regression analyses were run with BMI as the dependent variable and five independent variables including the total BNSS score and four factors that might make an independent prediction: gender, age, total antipsychotic equivalent dose and positive symptoms total score (Pos_PANSS). These four variables were selected due to gender differences in the distribution of BMI in the general population and known associations between age, medication and weight gain in schizophrenia (Cooper et al., 2016). Severity of positive symptoms was also included as patients with more severe symptoms are often treated with higher 
doses of medication, which has been known to have an association with more secondary negative symptoms (i.e. sedation or blunted affect) (Kirkpatrick \& Galderisi, 2008).

As secondary aims, we explored the prediction of the two primary factors of the BNSS (emotional life and motivation/pleasure) on BMI and whether different measures of negative symptoms (PANSS and EMSLEY) provided the same results.

All analyses were performed using SPSS v21.0 for Mac (IBM Inc.) and significance threshold was set at $\mathrm{p}<0.05$.

\section{Results}

From the initial 134 subjects, 66 were on clozapine or olanzapine treatment. Of those, three were excluded due to clozapine-aripiprazole combination. One additional participant (male) was excluded due to being overtly underweight, with a BMI score $<18$, which raised doubts about medication compliance. The final sample included 62 volunteers. Participants were recruited from the four participant centers: Cambridge (31), HMar (12), HClinic (11) and Oviedo (8). Sample socio-demographic and relevant clinical factors are presented in Table 1.

\subsection{Association between BMI and negative symptoms severity (measured with BNSS total} score)

Zero-order bivariate correlations showed a significant negative correlation between BMI and BNSS ( $r=-.31 ; p=.015$; Figure 1), such that lower negative symptoms were associated with higher BMI. The regression model (Table 2), which accounted for 19\% of the variance, was significant, $F(5,56)=2.57, p=.037$. Both negative symptoms (BNSS total score; $B=-.37, t=-$ 2.83, $p=.007)$ and age $(B=-.26, t=-2.00, p=.049)$ significantly predicted BMI. No other variable made significant independent predictions. 


\subsection{Association between BMI and BNSS subscales}

Here, we aimed to identify which specific negative symptoms predict BMI. For this set of analyses, we used the same model of multiple regression analysis as above, but also included Emotional expressivity and Motivation and Pleasure subscales as independent variables (separately). Both variables could not be used in the same model, due to the high colinearity between them $(r=.685 ; p<.001)$.

As shows Table 2, the first model (including Emotional expressivity) was non-significant $(p=0.91)$. However, the second model (including Motivation and Pleasure), which accounted for $18 \%$ of the variance, was significant $F(5,56)=2.43, p=.046$. Both motivation/pleasure $(B=-$ $.36, t=-2.71, p=.009)$ and age $(B=-.27, t=-2.09, p=.041)$ were significant predictors of BMI, whereas gender $(p=.441)$, medication equivalents $(p=.115)$ and positive symptoms $(p=.933)$ were not.

Specifically, Motivation and Pleasure includes three subscales of negative symptoms: anhedonia (items 1, 2, 3), asociality (items 5, 6) and avolition (items 7, 8). Using zero-order bivariate correlations, we found that BMI negatively correlated with anhedonia $(r=-.254 ; p=.046)$ and asociality $(r=-.253 ; p=.048)$, but not avolition $(r=-.169 ; p=.188)$, although significance levels would not survive when correcting for multiple comparisons.

\subsection{Association between BMI and PANSS negative symptoms subscales}

In our sample, the BNSS and Neg_PANSS were highly associated $(r=.908, p<.001)$. However, Neg_PANSS and BMI failed to reach significance $(r=-.224, p=.080)$, and a multiple regression analysis (using the same variables as above, except with Neg_PANSS as an independent variable) resulted in a non-significant model $(p=.156)$. Neg_PANSS did not significantly predict $\operatorname{BMI}(p=.055)$. 
Emsley PANSS highly correlated with BNSS $(r=.873, p<.001)$ and negatively correlated with BMI $(r=-.31 ; p=.014)$. The multiple regression model using Emsley_PANSS was significant $\left.\mathrm{F}(5,56)=2.52, p=.040, R^{2}=.18\right)$ and both Emsley_PANSS $(B=-.370, p=.007)$ and age $(B=-.256$, $p=.049)$ were significant predictors.

\subsection{Alternative analyses}

Using patients only treated with clozapine $(n=54)$ or with three BNSS components instead of two, the results did not change (data not shown).

We also re-analysed our data using the Peralta and Cuesta's formula (1994) and found that the pattern of results did not change. We found statistical significance between the negative symptom dimension and body mass index $(\mathrm{r}=-0.30, p=0.02)$. The multiple regression model using this formula was also significant $F(5,56)=2.32, p=0.05, \mathrm{R}^{2}=0.17$ and the Peralta and Cuesta's factor was a significant predictor of BMI $(B=-0.21, p=0.02)$.

\section{Discussion}

Contrary to our initial hypothesis, and other previous studies (Koga \& Nakayama, 2005)' (Janney et al., 2013), we found that lower negative symptoms were associated with higher BMI in patients with chronic schizophrenia prescribed with either clozapine or olanzapine treatment. This was primarily driven by lower anhedonia and asociality levels. This finding, if replicated, might be relevant when designing weight control interventions in patients with chronic schizophrenia treated with these medications, and possibly other antipsychotics.

Our study replicates the only study to date reporting that lower levels of negative symptoms are associated with higher BMI in chronic schizophrenia patients (Chen et al., 2014). However, opposite to previous studies with larger sample sizes (Chen et al., 2014; Gurpegui et al., 2012), 
we did not find any significant association between BMI and the total scores on the PANSS. This is likely due to some confounding factors related to outpatient's habits, such as exercise, lifestyle or economic status (Atmaca et al., 2007). However, our study is the first to use more sophisticated measures of negative symptoms, which arguably increases the validity of our results. Interestingly, it has been reported that patients under treatment with clozapine or olanzapine, the antipsychotics most related with weight gain, have been described to improve symptomatology associated with weight gain (Czobor et al., 2002). Noteworthy, our results were observed when using the BNSS, and not the commonly used Neg_PANSS score. This suggests that a more accurate scale for measuring negative symptoms is required to find these associations. Indeed, we aimed to confirm this by using the Emsley PANSS score, which is a PANSS scale derivate using a factor analysis, and therefore a more precise score for negative symptoms rather than the classical negative subscale from the PANSS. Furthermore, some of our results failed to reach significance, but were marginal. Future studies with larger samples (and more statistical power) are required to confirm if these effects are present.

We suggest that the role of anhedonia and motivation and their influence on reward processing, particularly in related to food rewards, are key factors for interpreting our results. Negative symptoms are associated with abnormal reward response (Kirschner et al., 2016) and anhedonia severity has been linked to decreased anterior cingulate and medial frontal cortical activation during task-related motivation (Segarra et al., 2016). Brain mechanisms associated with reward have also been studied in metabolic science (Ziauddeen et al., 2015), matching hedonic experience and motivation for food. More interestingly, the literature in psychosis has shown that enhanced reward activity is associated with higher BMI. Furthermore, a recent study showed that drug-naive patients showed higher anticipation during a task of reward activity in the right putamen after amisulpride treatment, and experienced greater weight gain (Nielsen et al., 2016). To replicate the role of the reward system in weight gain, it has been shown that 
administering low-dose olanzapine in healthy volunteers (Mathews et al., 2012) enhances reward activity in the dorsal striatum and increases eating behavior.

One possible interpretation is that a preserved hedonic experience in patients with chronic schizophrenia could be preventative for losing weight. We previously found that the main determinant of well-being perception in clozapine-treated patients was the presence of positive symptoms and not physical health (Brown et al., 2016). A plausible option is that food is a widely available reward cue that allows experiencing pleasure in those patients with a preserved reward system. Conversely, reduced hedonic experience and poor motivation might be linked to less food preparation (i.e. shopping) and eating necessary for intact daily functioning. Prioritizing an immediate reward such as food, especially one for the few available in this specific population over the executive control for delayed reward (i.e. to avoid metabolic complications to extend lifespan) might hamper successful interventions.

Indeed, in non-scare food environments such as Western countries, overconsumption is thought to be driven by a more hedonic form of eating (Zheng \& Berthoud, 2008). In mice, separate basal ganglia circuitries mediated the hedonic and nutritional actions of sugar (Tellez et al., 2016). It is also important to note that there is a more limited range of hedonic experiences reported by patients with schizophrenia, as social isolation, reduced sex life and unemployment are highly prevalent (Generator, 2014). If so, these factors should be taken into account during weight intervention strategies as, to date, pharmacological and non pharmacological interventions for weight control in patients with psychosis tend to fail (Speyer et al., 2016) or are of little clinical relevance and benefit (Daumit et al., 2013, Zimbron et al., 2016). Therefore, new interventions might need to challenge attitudes towards long-term consequences of poor physical health. If replicated, our results might help better design interventions for patients that might not consider physical health as a priority, or at least to a lesser extent than in the general population. 
Our study has some limitations. Firstly, no specific assessments of attitude toward physical health or measures of pleasure associated with eating were collected. Secondly, our sample size is relatively modest and our results require replication and extension in the same population. However, our study was conducted in four different sites in order to avoid sample selection bias. Another constraint is the cross-sectional design and the inherent limitation of establishing a cause-effect relationship, which warrants further research for understanding the casual role of antipsychotic medication on weight gain. Lastly, we did not collect measures of confounding factors that are likely related to BMI in schizophrenia. For this reason, more detailed studies with larger samples are needed to investigate the possible associations between negative symptoms and body mass index taking into account such confounds.

In conclusion, this study explored the prediction of negative symptoms on BMI in patients with schizophrenia prescribed with clozapine and/or olanzapine treatment. Our findings suggest that less negative symptoms severity, especially lower anhedonia and asociality symptom severity, predict increased BMI. These results might help to design more personalized interventions for patients with chronic schizophrenia, which could explore the subjective experience of eating or the level of insight or even interest about long-term physical health consequences. 


\section{Acknowledgments}

We are extremely grateful to all of the participants that took part in this study.

This study was supported by the Spanish Ministry of Science and Education, the Spanish Ministry of Economic Affairs and Competitiveness, ISCIII-General Evaluation Branch, by the Centro de Investigación Biomédica en Red de Salud Mental (CIBERSAM) and by the Secretaria d'Universitats i Recerca del Departament d'Economia i Coneixement, Government of Catalonia. 


\section{Role of funding source}

Some team members have received different sources of funding that have indirectly supported this work. None of the sources of funding have had any input in the analysis or the writing of this manuscript.

This work was supported by the Government of Catalonia, Secretaria d'Universitats i Recerca del Departament d'Economia i Coneixement (2014SGR441), with the grants FI-DGR-2013 Contract of the Agència de Gestió d'Ajuts Universitaris i de Recerca (AGAUR) (2015 FI_B2 00100) and from Fundació Bosch Gimpera (FBG) within the RETOS COLABORACIÓN 2015, funded by the Spanish Ministry of Economic Affairs and Competitiveness (RTC-20153440-1) to G. Mezquida. Dr. Bernardo has been supported by research funding from the Spanish Ministry of Health, the Spanish Ministry of Science and Education, the Spanish Ministry of Economic Affairs and Competitiveness, Centro de Investigación Biomédica en Red de Salud Mental (CIBERSAM), by Secretaria d'Univesritat i Recerca del Departament d'Economia i Coneixement (2014SGR441), Foundation European Group for Research In Schizophrenia (EGRIS), and the 7th Framework Program of the European Union. Dr. GarciaRizo is supported by the PI14/00753 project, integrated into the State Plan of Scientific and Technical Research and Innovation 2013-2016 and co-financed by the ISCIII-General Evaluation Branch and the European Regional Development Fund (ERDF). Dr. Bobes is supported by the Spanish Ministry of Health, the Spanish Ministry of Science and Education, the Spanish Ministry of Economic Affairs and Competitiveness and CIBERSAM. Dr. Paz-Portilla has been also supported by the European Comission, ISCIII-General Evaluation Branch and CIBERSAM. Dr. Savulich is funded by Eton College and the Wallitt Foundation. Dr. Fernandez-Egea is supported, in part, by the NIRH-Biomedical Research Center, Cambridge. 


\section{Conflitct of interest}

Dr. Bernardo has been a consultant or received grant/research support and honoraria from, and been on the speakers/advisory board of AB-Biotics, Adamed, Almirall, Amgen, Boehringer, Eli Lilly, Ferrer, Forum Pharmaceuticals, Gedeon, Hersill, Janssen-Cilag, Lundbeck, Otsuka, Pfizer, Roche and Servier. Dr. Mané has received financial support to attend meetings, travel support, and served as a speaker from Otsuka and Janssen Cilag. A. Toll has received financial support to attend meetings and travel support from Otsuka, Janssen Cilag, Lundbeck and Pfizer. Dr. Bobes received research grants and served as consultant, advisor or speaker for the companies: AB-Biotics, Adamed, Almirall, AstraZeneca, Bristol-Myers Squibb, Ferrer, GlaxoSmith-Kline, Hoffman La Roche, Janssen-Cilag, Lilly, Lundbeck, Merck, Novartis, Organon, Otsuka, Pfizer, Pierre-Fabre, Sanofi-Aventis, Servier and Shering-Plough and Shire. Dr. Garcia-Portilla has been a consultant and has received honoraria/grants from Alianza OtsukaLundbeck, Janssen-Cilag, Lilly, Lundbeck, Otsuka, Pfizer, Servier, Roche and Rovi. Dr. Fernandez-Egea, Dr. Garcia-Alvarez, Dr. Savulich, and Ms. Mezquida declare no conflicts of interest. 


\section{References}

Addington, D., Addington, J., Schissel, B., 1990. A depression rating scale for schizophrenics. Schizophr. Res. 3, 247-251.

Atmaca, M., Tezcan E., Ustundag, B., 2007. Plasma nitric oxide and leptin values in patients with olanzapine-induced wight gain. J. Psychiatr. Res. 41, 74-79.

Brown, J.E.H., Mezquida, G., Fernandez-Egea, E., 2016. Well-being in clozapine-treated schizophrenia patients: The significance of positive symptoms. Compr. Psychiatry 68, 140-46.

Bushe, C.J., Slooff, C.J., Haddad, P.M., Karagianis, J.L., 2013. Weight change by baseline BMI from three-year observational data: findings from the Worldwide Schizophrenia Outpatient Health Outcomes Database. J. Psychopharmacol. 27, 358-365.

Cooper, S.J., Reynolds, G.P., Barnes, T., England, E., Haddad, P.M., Heald, A., Holt, R., Lingford-Hughes, A., Osborn, D., McGowan, O., Patel, M.X., Paton, C., Reid, P., Shiers, D., Smith, J., 2016. BAP guidelines on the management of weight gain, metabolic disturbances and cardiovascular risk associated with psychosis and antipsychotic drug treatment. J. Psychopharmacol. 30, 717-748.

Czobor, P., Volavka, J., Sheitman, B., Lindenmayer, J.-P., Citrome, L., McEvoy, J., Cooper, T. B., Chakos, M., Lieberman, J.A., 2002. Antipsychotic-induced weight gain and therapeutic response: a differential association. J. Clin. Psychopharmacol. 22, 244-251.

Chen, S.F., Hu, T.M., Lan, T.H., Chiu, H.J., Sheen, L.Y., Loh, E.W., 2014. Severity of psychosis syndrome and change of metabolic abnormality in chronic schizophrenia patients: Severe negative syndrome may be related to a distinct lipid pathophysiology. Eur. Psychiatr. 29, 167-171.

Daumit, G.L., Dickerson, F.B., Wang, N.-Y., Dalcin, A., Jerome, G.J., Anderson, C.A.M., Young, D.R., Frick, K.D., Yu, A., Gennusa, J.V., Oefinger, M., Crum, R.M., Charleston, J., Casagrande, S.S., Guallar, E., Goldberg, R.W., Campbell, L.M., Appel, L.J., 2013. A behavioral weight-loss intervention in persons with serious mental illness. N. Engl. J. Med. 368(17), 1594-1602

Emsley, R., Rabinowitz, J., Torreman, M., 2003. The factor structure for the Positive and Negative Syndrome Scale (PANSS) in recent-onset psychosis. Schizophr. Res. 61, 47-57.

Fernandez-Egea, E., Miller, B., Garcia-Rizo, C., Bernardo, M., Kirkpatrick, B., 2011. Metabolic effects of olanzapine in patients with newly diagnosed psychosis. J. Clin. Psychopharmacol. 31, 154-9.

Garcia-Portilla, M.P., Garcia-Alvarez, L., Mané, A., Garcia-Rizo, C., Sugranyes, G., Bergé, D., Bernardo, M., Fernández-Egea, E., Bobes, J., 2015. The negative syndrome of schizophrenia: Three-underlying components are better than two. Schizophr. Res. 166(1-3), $115-118$

Gardner, D.M., Murphy, A.L., O'Donnell, H., Centorrino, F., Baldessarini, R.J., 2010. International consensus study of antipsychotic dosing. Am. J. Psychiatry 167, 686-693.

Generator, N.E.W., 2014. Psychosis and schizophrenia in adults: treatment and management (GC 178). NICE National Institute for Health and Care Excellence, 1-54.

Gurpegui, M., Martínez-Ortega, J.M., Gutiérrez-Rojas, L., Rivero, J., Rojas, C., Jurado, D., 2012. Overweight and obesity in patients with bipolar disorder or schizophrenia compared with a non-psychiatric sample. Prog. Neuropsychopharmacol. Biol. Psychiatry 37, 169-75.

Hermes, E., Nasrallah, H., Davis, V., Meyer, J., McEvoy, J., Goff, D., Davis, S., Stroup, T.S., Swartz, M., Lieberman, J., Rosenheck, R., 2011. The association between weight change and symptom reduction in the CATIE schizophrenia trial. Schizophr. Res. 128, 166-170. 
Janney, C.A., Ganguli, R., Richardson, C.R., Holleman, R.G., Tang, G., Cauley, J.A., Kriska, A.M., 2013. Sedentary behavior and psychiatric symptoms in overweight and obese adults with schizophrenia and schizoaffective disorders (WAIST Study). Schizophr. Res. 145, 63-68.

Kay, S.R., Fiszbein, A., Opler, L.A., 1987. The positive and negative syndrome scale (PANSS) for schizophrenia. Schizophr. Bull. 13, 261-276.

Kirkpatrick, B., Galderisi, S., 2008. Deficit schizophrenia: an update. World Psychiatry 7, 143147.

Kirkpatrick, B., Strauss, G.P., Nguyen, L., Fischer, B.A., Daniel, D.G., Cienfuegos, A., Marder, S.R., 2011. The brief negative symptom scale: psychometric properties. Schizophr. Bull. 37, 300-305.

Kirschner, M., Hager, O.M., Bischof, M., Hartmann-Riemer, M.N., Kluge, A., Seifritz, E., Tobler, P.N., Kaiser, S., 2016. Deficits in context-dependent adaptive coding of reward in schizophrenia. NPJ schizophrenia 2, 16020.

Koga, M., Nakayama, K., 2005. Body weight gain induced by a newer antipsychotic agent reversed as negative symptoms improved. Acta Psychiatr. Scand. 112, 75-77.

Leucht, S., Cipriani, A., Spineli, L., Mavridis, D., Orey, D., Richter, F., Samara, M., Barbui, C., Engel, R.R., Geddes, J.R., Kissling, W., Stapf, M.P., Lassig, B., Salanti, G., Davis, J. M. 2013a. Comparative efficacy and tolerability of 15 antipsychotic drugs in schizophrenia: a multiple-treatments meta-analysis. Lancet 382, 951-962.

Mané, A., García-Rizo, C., Garcia-Portilla, M. P., Bergé, D., Sugranyes, G., Garcia-Alvarez, L., Bernardo, M., Bobes, J. \& Fernandez-Egea, E. 2014. Spanish adaptation and validation of the Brief Negative Symptoms Scale. Compr. Psychiatry 55, 1726-1729.

Mathews, J., Newcomer, J.W., Mathews, J.R., Fales, C.L., Pierce, K.J., Akers, B.K., Marcu, I., Barch, D. M. 2012. Neural correlates of weight gain with olanzapine. Arch. Gen. Psychiatry $69,1226-1237$.

Nielsen, M.Ø., Rostrup, E., Wulff, S., Glenthøj, B., Ebdrup, B.H., 2016. Striatal Reward Activity and Antipsychotic-Associated Weight Change in Patients With Schizophrenia Undergoing Initial Treatment. JAMA Psychiatry 73, 121-28.

Peralta, V., Cuesta, M.J., 1994. Psychometric properties of the positive and negative syndrome scale (PANSS) in schizophrenia. Psychiatry Res. 53, 31-40.

Pérez-Iglesias, R., Martínez-García, O., Pardo-Garcia, G., Amado, J.A., Garcia-Unzueta, M. T., Tabares-Seisdedos, R., Crespo-Facorro, B., 2014. Course of weight gain and metabolic abnormalities in first treated episode of psychosis: the first year is a critical period for development of cardiovascular risk factors. Int. J. Neuropsychopharmacol. 17, 4151.

Reynolds, G.P., 2012. Pharmacogenetic Aspects of Antipsychotic Drug-induced Weight Gain A Critical Review. Clin. Psychopharmacol. Neurosci. 10, 71-77.

Royal College of Psychiatrists, 2014. Report of the Second Round of the National Audit of Schizophrenia (NAS). Healthcare Quality Improvement Partnership, London,

Segarra, N., Metastasio, A., Ziauddeen, H., Spencer, J., Reinders, N.R., Dudas, R. B., Arrondo, G., Robbins, T.W., Clark, L., Fletcher, P.C., Murray, G.K., 2016. Abnormal Frontostriatal Activity During Unexpected Reward Receipt in Depression and Schizophrenia: Relationship to Anhedonia. Neuropsychopharmacology 41, 2001-2010.

Simon, V., van Winkel, R., De Hert, M., 2009. Are weight gain and metabolic side effects of atypical antipsychotics dose dependent? A literature review. J. Clin. Psychiatry 70, 1041-1050.

Speyer, H., Christian Brix Nørgaard, H., Birk, M., Karlsen, M., Storch Jakobsen, A., Pedersen, K., Hjorthøj, C., Pisinger, C., Gluud, C., Mors, O., Krogh, J., Nordentoft, M., 2016. The CHANGE trial: no superiority of lifestyle coaching plus care coordination plus treatment as usual compared to treatment as usual alone in reducing risk of cardiovas- 
cular disease in adults with schizophrenia spectrum disorders and abdominal obesity. World Psychiatry 15, 155-165.

Strauss, G.P., Hong, L.E., Gold, J.M., Buchanan, R.W., McMahon, R.P., Keller, W.R., Fischer, B.A., Catalano, L.T., Culbreth, A.J., Carpenter, W.T., Kirkpatrick, B., 2012. Factor structure of the Brief Negative Symptom Scale. Schizophr. Res. 142, 96-98.

Tellez, L. A., Han, W., Zhang, X., Ferreira, T. L., Perez, I. O., Shammah-Lagnado, S. J., van den Pol, A. N. \& de Araujo, I. E. 2016. Separate circuitries encode the hedonic and nutritional values of sugar. Nature Neuroscience 19:465-70.

Zheng, H., Berthoud, H.-R., 2008. Neural systems controlling the drive to eat: mind versus metabolism. Physiology (Bethesda) 23, 75-83.

Ziauddeen, H., Alonso-Alonso, M., Hill, J.O., Kelley, M., Khan, N.A., 2015. Obesity and the neurocognitive basis of food reward and the control of intake. Adv. Nutr. 6, 474-486.

Ziauddeen, H., Garcia-Rizo, C., Bernardo, M., Kirkpatrick, B., Ozanne, S.E., Jones, P.B., Fernandez-Egea, E., 2016. Association of birth weight and the development of antipsychotic induced adiposity in individuals with treatment resistant schizophrenia. Eur. Neuropsychopharmacol. 26, 972-78.

Zimbron, J., Khandaker, G.M., Toschi, C., Jones, P.B., Fernandez-Egea, E., 2016. A systematic review and meta-analysis of randomised controlled trials of treatments for clozapineinduced obesity and metabolic syndrome. Eur. Neuropsychopharmacol. 26, 1353-1365.

Figure 1. Association between negative symptom severity and BMI. 